\title{
Isolating critical cases for reciprocals using integer factorization
}

\author{
John Harrison \\ Intel Corporation, JF1-13 \\ $2111 \mathrm{NE} 25$ th Avenue \\ Hillsboro OR, USA \\ johnh@ichips.intel.com
}

\begin{abstract}
One approach to testing and/or proving correctness of a floating-point algorithm computing a function $f$ is based on finding input floating-point numbers a such that the exact result $f(a)$ is very close to a "rounding boundary", i.e. a floating-point number or a midpoint between them. In the present paper we show how to do this for the reciprocal function by utilizing prime factorizations. We present the method and show examples, as well as making a fairly detailed study of its expected and worst-case behavior. We point out how this analysis of reciprocals can be useful in analyzing certain reciprocal algorithms, and also show how the approach can be trivially adapted to the reciprocal square root function.
\end{abstract}

\section{Background}

Suppose we have a floating-point algorithm computing a function that approximates a true mathematical function $f: \mathbb{R} \rightarrow \mathbb{R}$. For example, consider the following algorithm for the Intel ${ }^{\circledR}$ Itanium ${ }^{\circledR}$ architecture designed to compute a floating-point square root $\sqrt{a}$ using an initial reciprocal square root approximation followed by a sequence of fused multiply-adds. (In the actual implementation, the initial approximation instruction deals with special cases including $a=0$.)

$$
\begin{array}{lll}
\text { 1. } & y_{0}=\operatorname{frsqrta}(a) & \\
\text { 2. } & H_{0}=\frac{1}{2} y_{0} & S_{0}=a y_{0} \\
\text { 3. } & d_{0}=\frac{1}{2}-S_{0} H_{0} & \\
\text { 4. } & H_{1}=H_{0}+d_{0} H_{0} & S_{1}=S_{0}+d_{0} S_{0} \\
\text { 5. } & d_{1}=\frac{1}{2}-S_{1} H_{1} & \\
\text { 6. } & H_{2}=H_{1}+d_{1} H_{1} & S_{2}=S_{1}+d_{1} S_{1} \\
\text { 7. } & d_{2}=\frac{1}{2}-S_{2} H_{2} & e_{2}=a-S_{2} S_{2} \\
\text { 8. } & H_{3}=H_{2}+d_{2} H_{2} & S_{3}=S_{2}+e_{2} H_{2} \\
\text { 9. } & e_{3}=a-S_{3} S_{3} & \\
\text { 10. } & S=S_{3}+e_{3} H_{3} & \\
& &
\end{array}
$$

If an algorithm is, like this one, implemented by composing basic floating-point operations (rather than, say, some more complicated analysis of bit-patterns), then the value computed can usually be represented as the result of rounding some approximation $f^{*}(x) \approx f(x)$, the value before the final rounding. In this case, the final $S$ results from rounding the exact value $\mathrm{S}_{3}+e_{3} \mathrm{H}_{3}$.

The algorithm will therefore round correctly for all inputs $x$ such that $f^{*}(x)$ and $f(x)$ round to the same number (for all the rounding modes under consideration). In the concrete square root example, this means that $\sqrt{a}$ and $S_{3}+e_{3} H_{3}$ should always round the same way.

A sufficient condition for equivalent rounding behavior is that the two values $f^{*}(x)$ and $f(x)$ should never be separated by a rounding boundary, i.e. a floating-point number (for directed rounding) or a midpoint (for round-tonearest). That is, there is never a rounding boundary $m$ with $f(x) \leq m \leq f^{*}(x)$ or $f^{*}(x) \leq m \leq f(x)$, unless $f^{*}(x)=f(x)$. (Not quite a necessary condition in the round-to-nearest mode since if one is exactly equal to the rounding boundary and the other on the "right" side, the correct result will be obtained.) This is usually hard to establish by analytic reasoning. However, it is usually easy to establish some sort of relative error bound $\epsilon$ such that:

$$
\left|f^{*}(x)-f(x)\right| \leq \epsilon|f(x)|
$$

Therefore, misrounding can occur only when

$$
|f(x)-m| \leq \epsilon|f(x)|
$$

It is therefore interesting for purposes of both testing and proving correctness to deliberately concoct test points $x$ to make the relative distance from a rounding boundary $|f(x)-m| /|f(x)|$ as small as possible. Indeed, irrespective of the details of the algorithms we are concerned with, these test points might be expected to display greatest sensitivity to the accuracy of $f^{*}(x)$ and so show up errors most easily. 
For some basic algebraic functions, such special $x$ can be found analytically using number-theoretic techniques $[14,11]$, in such a way that the very worst examples (having the smallest relative distance from a rounding boundary) are isolated. For transcendental functions, this is more difficult, but one can still generate good cases by exploiting local linearity and solving congruences. For double-precision it is feasible, though costly, to isolate the very worst examples [6].

One use of the points so obtained is to test floating-point functions. Indeed, Parks [11] reports that such testing exposed a bug in a commercial microprocessor. A more ambitious goal, realized for square root algorithms by Cornea [1], is to isolate a sufficiently large set of points that the correct behavior of the algorithm on these, in conjunction with an analytical proof that covers all other cases, gives a complete correctness proof of the algorithm in all cases. For example, if we can prove analytically that for all floatingpoint numbers $x$ we have:

$$
\left|f^{*}(x)-f(x)\right| \leq \epsilon|f(x)|
$$

and that some set $S_{\epsilon}$ contains all points $x$ where $\mid m-$ $f(x)|\leq \epsilon| f(x) \mid$ for some rounding boundary $m$, the correctness of the algorithm in all cases is equivalent to the correctness just for the points in $S_{\epsilon}$. If such sets can be found easily and they are not too large, this gives a very effective methodology for proofs of algorithms. The goal of this paper is to show how to isolate such special cases for the reciprocal (and reciprocal square root) function and demonstrate their applicability in such correctness proofs of algorithms.

\section{Critical cases for quotient and reciprocal}

We will in what follows consider a single floating-point format with precision $p$, which contains all the floatingpoint numbers concerned and is also the destination format for the result. We also ignore the possibility of overflow and underflow in computation sequences. This keeps the presentation simpler and accords well with the intended applications where all input numbers are double-extended and additional exponent range (but not precision) is available for intermediate computations. The results that follow can straightforwardly be refined for mixed-precision applications.

It's instructive to examine the problem for the general case of quotients, and then contrast the restriction to the reciprocal. In general, we seek floating-point numbers $x$ and $y$ such that $x / y$ lies close to some $w$ that is either itself a floating-point number or a midpoint between two floatingpoint numbers. Without loss of generality, we can assume:

$$
\begin{array}{ll}
x=2^{e_{x}} a & 2^{p-1} \leq a<2^{p} \\
y=2^{e_{y}} b & 2^{p-1} \leq b<2^{p} \\
w=2^{e_{w}} m & 2^{p} \leq m<2^{p+1}
\end{array}
$$

where $p$ is the floating-point precision and $a, b$ and $m$, as well as the various $e_{i}$, are integers. Note that even values of $m$ correspond to floating-point numbers and odd values correspond to midpoints. We are interested in how small the relative difference $|x / y-w| /|x / y|$ can become. This relative difference can be rewritten as:

$$
\frac{|x / y-w|}{|x / y|}=|1-w y / x|=\left|1-2^{-q} m b / a\right|
$$

where $q=e_{x}-\left(e_{w}+e_{y}\right)$, and so

$$
\frac{\left|m b-2^{q} a\right|}{2^{q} a}
$$

Given the ranges of the values $a, b$ and $m$, we have

$$
2^{2 p-1} \leq m b<2^{2 p+1}
$$

and

$$
2^{q+p-1} \leq 2^{q} a<2^{q+p}
$$

It turns out that the only interesting cases are when $q=p$ or $q=p+1$. For if $q \leq p-1$ then $q+p \leq 2 p-1$ so we have

$$
2^{q} a \leq 2^{q}\left(2^{p}-1\right)<2^{2 p-1} \leq m b
$$

(remember that the values $a, b$ and $m$ are integers so when $<2^{r}$ they are actually $\left.\leq 2^{r}-1\right)$ and so

$$
\frac{\left|m b-2^{q} a\right|}{2^{q} a} \geq 2^{q} /\left(2^{q} a\right)=1 / a>2^{-p}
$$

Similarly if $q=p+2$ we have:

$$
m b \leq\left(2^{p}-1\right)\left(2^{p+1}-1\right)<2^{2 p+1} \leq 2^{p+2} a<2^{2 p+2}
$$

and therefore

$\frac{\left|m b-2^{q} a\right|}{2^{q} a} \geq\left(2^{p+1}+2^{p}-1\right) /\left(2^{q} a\right)>2^{p+1} / 2^{2 p+2}=2^{-(p+1)}$

Finally, if $q \geq p+3$ then $2^{q} a>2 m b$ and so

$$
\frac{\left|m b-2^{q} a\right|}{2^{q} a}>1 / 2
$$

In all these cases, the distance is at least $2^{-(p+1)}$. Therefore, when seeking cases where the distance is of order $2^{-2 p}$ (for realistic $p$ ) we need only consider $q \in\{p, p+1\}$. This 
being the case, the denominator $2^{q} a$ is constrained to within a factor of 4 , so the essential problem is to find how small

$$
\left|m b-2^{q} a\right|
$$

can become for $q \in\{p, p+1\}$. Since the value is an integer, we can try to find small values by explicit consideration of the various possibilities in succession:

$$
\begin{aligned}
m b & =2^{p} a+1 \\
m b & =2^{p} a-1 \\
m b & =2^{p+1} a+1 \\
m b & =2^{p+1} a-1 \\
m b & =2^{p} a+2 \\
m b & =2^{p} a-2 \\
m b & =2^{p+1} a+2 \\
m b & =2^{p+1} a-2 \\
m b & =2^{p} a+3 \\
\ldots &
\end{aligned}
$$

It seems that the number of possible solutions of these equations is too large for this to be a practical approach. On the other hand, if we fix any one of the values $a, b$ and $m$, the problem becomes tractable. If we fix either $m$ or $b$ then the problem becomes a set of linear congruences (with additional range restrictions filtering the possible solution set), which are easy to solve. If we consider the special case of the reciprocal, then we fix $a=2^{p-1}$. This problem is also tractable, as we shall see, but has a somewhat different character. We just need to consider

$$
\begin{aligned}
& m b=2^{2 p-1}+\delta \\
& m b=2^{2 p}+\delta
\end{aligned}
$$

for successive small integers $\delta$. In fact, the situation is even better, because once again no small values can arise in the former case because of the range limitation, except for the trivial $m b=2^{2 p-1}$; the next case must be $\left(2^{p}+1\right) 2^{p-1}=$ $2^{2 p-1}+2^{p-1}$. So we need only be concerned with solutions to

$$
m b=2^{2 p}+\delta
$$

for integers $2^{p-1} \leq b<2^{p}$ and $2^{p} \leq m<2^{p+1}$. Indeed, for small $\delta$, it is easy to see that the two upper bounds imply the lower ones.

\section{Factorization distribution}

Our approach to the problem of finding all solutions to $m b=2^{2 p}+\delta$ (with $p$ and $\delta$ fixed) is quite straightforward. We find the prime factorization of $2^{2 p}+\delta$, and consider all possible ways of distributing these prime factors into two parts $m$ and $b$ subject to the appropriate range limitation $m<2^{p+1}$ and $b<2^{p}$. In general, we will refer to a factorization $n=a b$ of $n$ with $a<A$ and $b<B$ as an (A, B)-balanced factorization.

Consider, for illustration, the case $p=6$ and $\delta \in$ $\{ \pm 1, \pm 2, \pm 3\}$. In each case we find the prime factorization of $2^{2 p}+\delta$ :

$$
\begin{aligned}
& 2^{12}+1=17 \cdot 241 \\
& 2^{12}-1=3^{2} \cdot 5 \cdot 7 \cdot 13 \\
& 2^{12}+2=2 \cdot 3 \cdot 683 \\
& 2^{12}-2=2 \cdot 23 \cdot 89 \\
& 2^{12}+3=4099 \\
& 2^{12}-3=4093
\end{aligned}
$$

In the cases $2^{12}+1,2^{12}+2,2^{12}+3$ and $2^{12}-3$, the largest factor is already $>2^{p+1}=128$, so there is no possible distribution obeying the range restrictions. For $2^{12}-2$ there is exactly one such distribution:

$$
m \cdot b=89 \cdot(2 \cdot 23)=89 \cdot 46
$$

Note that the 'symmetrical' distribution is not admissible because $89>2^{p}$. For $2^{12}-1$, there are four possible distributions:

$$
\begin{aligned}
& m \cdot b=\left(3^{2} \cdot 13\right) \cdot(5 \cdot 7)=117 \cdot 35 \\
& m \cdot b=(3 \cdot 5 \cdot 7) \cdot(3 \cdot 13)=105 \cdot 39 \\
& m \cdot b=(7 \cdot 13) \cdot\left(3^{2} \cdot 5\right)=91 \cdot 45 \\
& m \cdot b=(5 \cdot 13) \cdot\left(3^{2} \cdot 7\right)=65 \cdot 63
\end{aligned}
$$

Note that the corresponding $m$ are all odd, and therefore represent midpoints. Thus, we can say that $|1 / y-w| \geq$ $4 / 2^{12}|1 / y|$ for any midpoint $w$ except in the cases where $y$ 's significand $b$ is in the set $\{35,39,45,46,63\}$; for $b=46$ we get a $2 / 2^{12}$ relative distance and for $35,39,45$ and 63 we get $1 / 2^{12}$. Since the above lists exhausts all $m$, even or odd, we see that $|1 / y-w| \geq 4 / 2^{12}|1 / y|$ for any floatingpoint number $w$, except for the special cases when $y$ is a power of 2 and so its reciprocal is exactly representable (i.e. $1 / y=w)$. 


\section{Implementation}

The implementation of the above idea is straightforward, given any reasonable programming language. We have used Objective CAML, a very high-level functional language that we have previously used extensively for implementation of theorem proving code:

http://www.ocaml.org/

This already has a multiprecision integer and rational function datatype available. It does not, however, have a built-in library for factoring numbers, and we did not want to write our own code for this operation - since the numbers can be as large as $2^{226}$ (for quad precision reciprocals), factorization is a non-trivial problem. We used the factoring code included in the PARI / GP system:

$$
\text { http://www.parigp-home.de/ }
$$

The documentation says:

factorint $(n,\{$ flag $=0\})$ : factors the integer $\mathrm{n}$ using a combination of the Shanks SQUFOF and Pollard Rho method (with modifications due to Brent), Lenstra's ECM (with modifi cations by Montgomery), and MPQS (the latter adapted from the LiDIA code with the kind permission of the LiDIA maintainers), as well as a search for pure powers with exponents $\leq 10$.

We are not experts in the topic of factorization, but have been quite impressed with how fast it usually factors numbers. Only for quad precision, when the numbers are of the order $2^{226}$, does it start to slow down noticeably. Rather than a strict primality test, the factors are only subjected to a strong probabilistic primality test. Therefore, out of paranoia, we have developed our own code to certify primality, by constructing prime certificates in the style of Pratt [12], appealing to Lucas's theorem. That is, to certify that each $p$ occurring in PARI/GP's factorization is prime, we show that there is a primitive root $a$ modulo $p$ such that $a^{p-1} \equiv 1$ $(\bmod p)$ but $a^{\frac{p-1}{q}} \not \equiv 1 \quad(\bmod p)$ for any prime factor $q$ of $p-1$. (The primitive root $a$ is found randomly, and the factors $q$ of $p-1$ are found by using PARI/GP's factorization recursively, certifying those factors as primes too.) This certification slows down the factorization process by a moderate amount, so we sometimes switch it off when experimenting.

Once we have the prime factors, we need to test all ways of distributing them over two numbers subject to range restrictions. As noted, we need only apply the upper range restrictions $m<2^{p+1}$ and $b<2^{p}$. Roughly, we just naively enumerate all possibilities. In order to cut off choice points as soon as possible, we start distributing from the largest prime factors, i.e. consider the prime factors $p_{1}^{\alpha_{1}} \cdot p_{2}^{\alpha_{2}} \cdots \cdots p_{k}^{\alpha_{k}}$ in decreasing order $p_{1}>p_{2}>\cdots>p_{k}$. We first consider all $\alpha_{1}+1$ ways of distributing $p_{1}^{\alpha_{1}}$ into two parts. If any of these distributions already violate the range restriction, they are abandoned. Otherwise, for each one, we consider the $\alpha_{2}+1$ ways of distributing $p_{2}^{\alpha_{2}}$, and so on. The algorithm is very straightforward to program recursively in OCaml.

It might be doubted whether such a naive distribution algorithm is acceptably efficient. At least it has been adequate to obtain some results quite quickly for the main precisions that interest us, $p \in\{24,53,63,113\}$. We first look at some of these results and then turn to a detailed performance analysis.

\section{Results}

Table 1 presents a small sample of the results obtained using the methods outlined above. For each of the four major precisions $p=24,53,64,113$, we list the 66 floatingpoint significands whose reciprocals are closest either to floating-point numbers or midpoints. This distance, as a multiple of the corresponding $2^{-2 p}$, is given in the ' $d$ ' columns. When, as often happens, several reciprocals have the same ' $d$ ' value we order them in decreasing order, and cut the table off on that basis. The asterisk means that the distance is from a floating-point number (and hence may be unimportant if we are concerned only with round-tonearest).

Larger lists for $d$ up to a few thousand can be generated for all these precisions without requiring more than a few days of runtime on a modern machine. And of course, it is trivial to parallelize the task since it consists of a separate subtask for each $d$ considered.

\section{Applications}

We can use the techniques set out above in the design and verification of algorithms for correctly rounded reciprocals. These might be substituted by the programmer, or by the compiler if it can recognize that in an expression $a / b$, the constant $a$ is guaranteed to be 1 . (This could be generalized to any power of 2.) For example, the following algorithm is normally used for double-extended precision division (precision $p=64$ ) on Intel $\AA^{\circledR}$ Itanium ${ }^{\circledR}$ processors.

$\begin{array}{lll}\text { 1. } & y_{0}=\operatorname{frcpa}(b) & \\ \text { 2. } & d=1-b y_{0} & q_{0}=a y_{0} \\ \text { 3. } & d_{2}=d d & d_{3}=d d+d \\ \text { 4. } & y_{1}=y_{0}+y_{0} d_{3} & d_{5}=d_{2} d_{2}+d \\ \text { 5. } & y_{2}=y_{0}+y_{1} d_{5} & r_{0}=a-b q_{0} \\ \text { 6. } & e=1-b y_{2} & q_{1}=q_{0}+r_{0} y_{2} \\ \text { 7. } & y_{3}=y_{2}+e y_{2} & r=a-b q_{1} \\ \text { 8. } & q=q_{1}+r y_{3} & \end{array}$




\begin{tabular}{|c|c|c|c|c|c|c|c|}
\hline \multicolumn{2}{|c|}{ Single precision } & \multicolumn{2}{|l|}{ Double precision } & \multicolumn{2}{|l|}{ Extended precision } & \multicolumn{2}{|l|}{ Quad precision } \\
\hline Mantissa & $d$ & Mantissa & $d$ & Mantissa & $d$ & Mantissa & $d$ \\
\hline $0 \times 800000$ & $0^{*}$ & $0 \times 10000000000000$ & $0^{*}$ & $0 \times 8000000000000000$ & $0^{*}$ & $0 \times 10000000000000000000000000000$ & $0^{*}$ \\
\hline OXFFFFFF & 1 & 0x1FFFFFFFFFFFFF & 1 & OXFFFFFFFFFFFFFFFF & 1 & 0x1FFFFFFFFFFFFFFFFFFFFFFFFFF & 1 \\
\hline $0 \times E E 01 F F$ & 1 & $0 \times 1 F F F F F F 8000001$ & 1 & $0 \times \mathrm{D} 6329033 \mathrm{D} 6329033$ & 1 & 0X1FFFFFFFFFFFFFE00000000000001 & 1 \\
\hline $0 \times \mathrm{xFC} 3237$ & 1 & 0x1FD8CD299E8D 79 & $2^{*}$ & 0xB7938C6947D97303 & 1 & 0x1B52F1BB6F8DC3F0D 920E2F 3D 449B & 1 \\
\hline $0 \times F O F F O F$ & 1 & $0 \times 1 F C 94266515 B C 9$ & $2^{*}$ & 0X99D0C 486A0FAD 481 & 1 & $0 \times 19 \mathrm{C} 1 \mathrm{ECF} 3420 \mathrm{D} 27 \mathrm{~F} 8729 \mathrm{BA} 7 \mathrm{E} 1 \mathrm{AB} 31 \mathrm{D}$ & 1 \\
\hline $0 \times F 02 A 3 B$ & 1 & OX1F739BD 4 59BEA2 & 2 & 0×989E556CADAC2D7F & 1 & 0x17ABDE305BAC595488190B 4AD 7657 & 1 \\
\hline $0 \times \mathrm{XF} 00 \mathrm{FF} 1$ & 1 & 0x1F65FAD23B0D 86 & 2 & 0x8E05E117D9E786D5 & 1 & 0x14367E6C7D1CD 9E2833D2900EE8D5 & 1 \\
\hline $0 \times E E 4 B C 5$ & 1 & 0x1EF7930608393E & 2 & OXFFFFFFFFFFFFFE & $4^{*}$ & 0X1FFBA28E4810FB56A9FDD85058227 & $2^{*}$ \\
\hline $0 \times \mathrm{EC} 7 \mathrm{EC} 7$ & 1 & 0x1EDA 43AEE3120F & $2^{*}$ & OXFFFFFFFE 00000002 & $4^{*}$ & 0X1FF0231E35F 73DFF14F 8 9AADF 10C2 & 2 \\
\hline $0 \times E 25473$ & 1 & $0 \times 1 \mathrm{ED} 31 \mathrm{~F} 284 \mathrm{BA} 183$ & $2^{*}$ & OXFFFFF $000007 \mathrm{FFFFE}$ & $4^{*}$ & 0X1FE5A1913A4EF 66DEF 762D8053282 & 2 \\
\hline $0 \times \mathrm{xE} 1368 \mathrm{~B}$ & 1 & 0X1E9A9473949BE 6 & 2 & OXFF $801 \mathrm{FFA} 00 \mathrm{FFE} 002$ & $4^{*}$ & 0X1FE1696E4EFFB 6A84655C0D432D 92 & 2 \\
\hline $0 \times E 05475$ & 1 & 0x1E8D517D09C5C2 & 2 & $0 \times \mathrm{FF} 007 \mathrm{FC} 01 \mathrm{FF} 007 \mathrm{FE}$ & $4^{*}$ & 0x1FDA070427995BB524AB4B13DC457 & $2^{*}$ \\
\hline $0 \times D E 86 A 9$ & 1 & 0x1E756F08DF1792 & 2 & 0XFE421D63446A3B34 & 4 & 0×1FAA42B2AE532A32F 819FE18EDEAF & $2^{*}$ \\
\hline $0 \times D C 23 D D$ & 1 & 0x1E4599DD926B71 & $2^{*}$ & 0XFDC1EAD583108905 & $4^{*}$ & 0×1F97117BE0A19F4B8279CEBB8A682 & 2 \\
\hline $0 \times \mathrm{D} 43 \mathrm{D} 43$ & 1 & 0x1E2 0ADBC $4078 \mathrm{~A} 2$ & 2 & 0XFC41DF1077C41DF3 & $4^{*}$ & 0x1F76B18346B7182CE92732C773FB2 & 2 \\
\hline $0 \times \mathrm{D} 25 \mathrm{D} 25$ & 1 & 0X1DE4A0D00FA9B2 & 2 & $0 \times F C 07 F F E 03 F F F 01 F E$ & $4^{*}$ & 0×1F742DB89E4A0B81D5A2FE647E4EB & $2^{*}$ \\
\hline OXDODDOD & 1 & 0X1DE441D5331432 & 2 & $0 \times \mathrm{FC} 07 \mathrm{~F} 01 \mathrm{FC} 07 \mathrm{~F} 01 \mathrm{FE}$ & $4^{*}$ & $0 \times 1 F 490212 \mathrm{CC} 8000000003 \mathrm{E} 92042599$ & $2^{*}$ \\
\hline $0 \times \mathrm{DDAC} 19$ & 1 & 0x1DA83EEDD 80267 & $2^{*}$ & 0XFBFC17DFE0BEFF 04 & 4 & 0X1F40436566B31BF 75C99DF 4 4F 291F & $2 *$ \\
\hline $0 \times \mathrm{x} 23 \mathrm{DC} 3$ & 1 & 0X1DA210DAEB138E & 2 & 0XFB2 0F $95555168 D 17$ & $4^{*}$ & 0X1F361A9D 4 98B669732A60AFCF 9461 & $2^{*}$ \\
\hline $0 \times \mathrm{C} 100 \mathrm{C} 1$ & 1 & 0X1D7F 8AC20F7A3F & $2^{*}$ & OXFB0089D7241D10FC & 4 & 0x1F25136B121FE2DD08B9975B8DBD2 & 2 \\
\hline $0 \times B 84 A 93$ & 1 & 0X1D7B72B82BAE23 & $2^{*}$ & 0XFA0BF7D05FBE82FC & 4 & 0X1F1DFB37ABDE $94 \mathrm{~B} 6800 \mathrm{C} 5550 \mathrm{CD} 152$ & 2 \\
\hline 0xAD1367 & 1 & 0x1D5B9032F086BE & 2 & 0XF $98 \mathrm{AF} 433 \mathrm{~A} 85 \mathrm{E} 62 \mathrm{BF}$ & $4^{*}$ & 0X1F182E16A52503DCEDEBC24CA2B4E & 2 \\
\hline 0XAB8BE1 & 1 & 0x1D5616F7BA44B9 & $2^{*}$ & 0XF96BA2 4DC930852A & $4^{*}$ & 0x1F140333F 6B5946A06272DBD508B7 & $2^{*}$ \\
\hline $0 \times A 6449 \mathrm{~F}$ & 1 & OX1C8ECFA282734B & $2^{*}$ & 0XF93AB02081C9D1D 6 & $4^{*}$ & 0x1F0DD51725F05CC5C752AA05A4311 & $2^{*}$ \\
\hline $0 \times A 24 \mathrm{CF} 7$ & 1 & $0 \times 1 \mathrm{C} 69 \mathrm{BF} 28 \mathrm{EBA} 166$ & 2 & 0XF912590F016D 6D0 4 & 4 & 0X1F001BEA0DE009CE597A0A8CE 4B02 & 2 \\
\hline 0xA0DDD 1 & 1 & $0 \times 1 \mathrm{C} 67 \mathrm{CF} 42 \mathrm{~F} 20 \mathrm{D} 11$ & $2^{*}$ & 0XF858A9FE5A20550D & $4^{*}$ & 0x1EC36516E1240A243EF66232D4BA7 & $2^{*}$ \\
\hline OX9BEAAF & 1 & $0 \times 1 C 4 D 3 A A B D 478 F 6$ & 2 & $0 \times F 84 \mathrm{CEE} 8 \mathrm{E} 701 \mathrm{FC} 266$ & $4^{*}$ & 0x1EBC 4C4507F 9CE8304761C8F 703D2 & 2 \\
\hline $0 \times 986799$ & 1 & $0 \times 1 C 2693$ DCF 34742 & 2 & 0XF774DD7F 912E1F54 & 4 & 0x1E9EDBD 04 7D1D813FB315AB469B2E & 2 \\
\hline $0 \times 909909$ & 1 & 0×1BEA3278B789D2 & 2 & 0XF744 4DFBF7B2 OEAC & 4 & 0x1E89A9332E4A8C84E2AA22A6DF7F 1 & $2^{*}$ \\
\hline $0 \times 8 \mathrm{EFA} 43$ & 1 & 0×1BB2278C9B2F 97 & $2^{*}$ & 0XF6F0243D8121FB7A & $4^{*}$ & 0x1E8119576512C73436A03607DCB9B & $2^{*}$ \\
\hline $0 \times 87 C C 45$ & 1 & 0x1B962F 9 EBB 9659 & $2^{*}$ & 0XF6640F754B4E709A & $4^{*}$ & 0×1E765D90D920CEEEBD7F 5E0E0BBA9 & $2^{*}$ \\
\hline $0 \times 869913$ & 1 & 0x1B227794E85702 & 2 & $0 \times F 39 \mathrm{~EB} 657 \mathrm{E} 24734 \mathrm{AC}$ & 4 & 0x1E5DF 4E7C4BB29C00588956 & $2^{*}$ \\
\hline $0 \times C A 6691$ & $2^{*}$ & 0x1B0FD7099EB189 & $2^{*}$ & 0XF 36EE790DE069D54 & 4 & 0X1E587973506590E472C4A72A35CF 1 & $2 *$ \\
\hline $0 \times A 1 E 58 F$ & $2^{*}$ & 0x1B0942AAAE0BD 3 & 2 & $0 \times \mathrm{XF} 363 \mathrm{~A} 464 \mathrm{E} 2 \mathrm{DCD} 8 \mathrm{~EB}$ & $4^{*}$ & 0X1E40DECFCF 36257C367CACDAD 3F 77 & $2^{*}$ \\
\hline OXFFFFFE & $4^{*}$ & $0 \times 1 A E 6849 \mathrm{E} 786 \mathrm{AD} 2$ & 2 & 0XF286AD 7943D 79434 & 4 & 0X1E2BE9D 384CE2D85FD8013E21ECF 2 & 2 \\
\hline $0 \times \mathrm{XFE} 002$ & $4^{*}$ & 0X1АBBEB8E009CE1 & $2^{*}$ & 0XEF 9DA1D 868469215 & $4^{*}$ & 0x1E15BB4DD7AA987E15487C533C649 & $2^{*}$ \\
\hline OXAAAAAC & 4 & 0x1AA7C88EE59082 & 2 & 0XEDF09CCC53942014 & 4 & 0x1E0C9181ECD $8418355 A 1 A 49887852$ & 2 \\
\hline $0 \times 8 \mathrm{C} 1284$ & 4 & 0x1A6F 41DAB98CB2 & 2 & 0XEDE $957 \mathrm{FFFFC} 485 \mathrm{AA}$ & $4^{*}$ & $0 \times 1 \mathrm{E} 0697 \mathrm{C} 8651 \mathrm{~B} 43 \mathrm{~A} 9309 \mathrm{DE} 9 \mathrm{E} 6 \mathrm{~F} 021 \mathrm{E}$ & 2 \\
\hline $0 \times 801001$ & $4^{*}$ & 0X1A2CE 4D 7478A0 6 & 2 & 0XEDE $95090 B 57 B 7 A 56$ & $4^{*}$ & 0x1DEE59C5D9CC8CB8613C8C6AD453F & $2 *$ \\
\hline $0 \times 800001$ & $4^{*}$ & 0x1A149BAD85DE72 & 2 & 0XEDBAA0 922AFB 6EAA & $4^{*}$ & 0×1DBA39CE33CA8DEF 599F 5DA2A534F & $2^{*}$ \\
\hline $0 \times 94 \mathrm{~F} 105$ & 5 & 0X1A0E795098FF63 & $2^{*}$ & 0XEC4B058D0F7155BC & 4 & 0x1DAC85098ABA5E144E44187FB5467 & $2^{*}$ \\
\hline $0 \times 92 A B A B$ & 5 & 0X1A0B8FFFFCBE8E & 2 & $0 \times \mathrm{XEC} 1 \mathrm{CA} 6 \mathrm{DB} 6 \mathrm{D} 7 \mathrm{BD} 444$ & 4 & 0x1D99C15392893EA6B5200AB3E8819 & $2^{*}$ \\
\hline $0 \times E 401 C 8$ & 8 & 0x19F142D24E1352 & 2 & 0XEB4 43F5A21FAD10E & $4^{*}$ & 0x1D983DBB99EAC $626064 \mathrm{~F} 81 \mathrm{D} 7 \mathrm{BF} 4 \mathrm{D} 2$ & 2 \\
\hline $0 \times E 071 \mathrm{~F} 9$ & $8^{*}$ & 0x19BD2D9FD24AD7 & $2^{*}$ & 0XEA6EE2D 972746 ED 1 & $4^{*}$ & 0x1D86CC4938A03D4525C152AB8505E & 2 \\
\hline $0 \times \mathrm{D} 6 \mathrm{D} 764$ & $8^{*}$ & 0x19B8D 7C084EE43 & $2^{*}$ & 0XEA4 0E197842DA6AF & $4^{*}$ & AD 5E05BA & 2 \\
\hline $0 \times \mathrm{D} 443 \mathrm{~F} 2$ & $8^{*}$ & $0 \times 199 \mathrm{E} 1 \mathrm{~B} 447 \mathrm{E} 99 \mathrm{C} 2$ & 2 & 0XE934A8E070ACB 65D & $4^{*}$ & 0x1D4D 79E1F152354E10F583D4A65C9 & $2^{*}$ \\
\hline $0 \times \mathrm{CFBA} 38$ & 8 & $0 \times 19939800033273$ & $2^{*}$ & $0 \times E 84 B D A 12 F 684 B D A 3$ & $4^{*}$ & 0×1D4CF 86C34F75247D8FA16202FA29 & $2^{*}$ \\
\hline $0 \times B 5 C 2 F 1$ & $8^{*}$ & 0x1975E059B82E 49 & $2^{*}$ & 0XE775FF 856986AE74 & 4 & 0×1D4562A76F 879A38EEE 86F 526D231 & $2^{*}$ \\
\hline $0 \times B 447 B C$ & $8^{*}$ & $0 \times 1960 A 45 D 1 A 71 E 6$ & 2 & 0XE6944AE6502F 8A22 & $4^{*}$ & 0x1D3168C71EC1068F69A433D0DF 9B7 & $2^{*}$ \\
\hline $0 \times A E 4 F 88$ & 8 & 0x19385F 4F 83B2B1 & $2^{*}$ & $0 \times E 5 C B 972 \mathrm{E} 5 \mathrm{CB} 972 \mathrm{E} 4$ & 4 & 0×1D26EDCA8F70B604EC3E7797A93A1 & $2^{*}$ \\
\hline $0 \times 9 A 5 F 6 E$ & $8^{*}$ & 0x190759A7F39561 & $2^{*}$ & 0XE597116BD81B26A3 & $4^{*}$ & 0x1D16B51A196CFFF2477078355A9AE & 2 \\
\hline $0 \times 988597$ & $8^{*}$ & $0 \times 18 \mathrm{~F} 187 \mathrm{FFFCE} 1 \mathrm{CF}$ & $2^{*}$ & $0 \times E 58 \mathrm{C} 38 \mathrm{D} 1342 \mathrm{FBE} 3 \mathrm{~A}$ & $4^{*}$ & 0X1D12CF093CC27703DA21DC7D 68CE7 & $2^{*}$ \\
\hline $0 \times 91 F E D C$ & $8^{*}$ & $0 \times 18 D F A 37 A 569 E 47$ & $2^{*}$ & 0XE58469F0234F72C4 & 4 & $0 \times 1 \mathrm{CEC} 5 \mathrm{ADBF} 01 \mathrm{E} 9685 \mathrm{CD} 487 \mathrm{AD} 8 \mathrm{~F} 3327$ & $2^{*}$ \\
\hline OXFFFFFD & 9 & $0 \times 1879574$ AF 5 FBB1 & 2 & $0 \times E 511 C 4648 \mathrm{E} 2332 \mathrm{C} 4$ & 4 & $0 \times 1 \mathrm{CE} 72221273 \mathrm{FE} 0035 \mathrm{FEC} 64 \mathrm{CBB} 3 \mathrm{DBF}$ & $2 *$ \\
\hline 0XA013D1 & 11 & $0 \times 184 \mathrm{~A} 12 \mathrm{EFEF} 626 \mathrm{E}$ & 2 & $0 \times E 3 F C 771 \mathrm{FE} 3 \mathrm{~B} 8 \mathrm{FF} 1 \mathrm{C}$ & 4 & 0X1CE 4C2D686D170738B 75E2AFECF 3E & 2 \\
\hline $0 \times \mathrm{F} 56 \mathrm{DA} 7$ & $12^{*}$ & $0 \times 18401 \mathrm{CBCDB} 5596$ & 2 & $0 \times E 3 C 845 B 18 B D 25 E C 6$ & $4^{*}$ & $0 \times 1 \mathrm{CD} 0 \mathrm{C} 2468 \mathrm{D} 84 \mathrm{~F} 6 \mathrm{ACF} 871 \mathrm{D} 5 \mathrm{E} 1 \mathrm{FCBA} 9$ & $2^{*}$ \\
\hline $0 \times 858376$ & $12^{*}$ & 0x181EFE51EAD722 & 2 & $0 \times E 318 \mathrm{DE} 3 \mathrm{C} 8 \mathrm{E} 6370 \mathrm{E} 4$ & 4 & 0×1CCB50FE 42CD1B95A59CA8AD 6EB99 & $2^{*}$ \\
\hline 0XCF7D 05 & 13 & $0 \times 1806 \mathrm{C} 89 \mathrm{FCB} 9452$ & 2 & 0XE301201062C997DE & $4^{*}$ & 0×1CABCCB01B54CE7E2A63A99B9D 9C2 & 2 \\
\hline $0 \times E E 5223$ & $14^{*}$ & $0 \times 17 \mathrm{~F} 52093014 \mathrm{~F} 0 \mathrm{E}$ & 2 & 0XE23B9711DCB88EE4 & 4 & 0X1C9ED 60CFD93F55F117571C3FDA0E & 2 \\
\hline $0 \times E 528 \mathrm{AB}$ & $14^{*}$ & $0 \times 17 D 93736 \mathrm{C} 115 \mathrm{FF}$ & $2^{*}$ & $0 \times E 231188 C 46231187$ & $4^{*}$ & $0 \times 1 \mathrm{C} 820 \mathrm{E} 19 \mathrm{C} 1 \mathrm{~A} 66 \mathrm{CE} 04 \mathrm{C} 62 \mathrm{~A} 562 \mathrm{E} 9111$ & $2^{*}$ \\
\hline $0 \times B F 3621$ & $14^{*}$ & 0x17A6B0778D60C1 & $2^{*}$ & $0 \times E 1 F 00785 \mathrm{C} 1 \mathrm{FF} 0 \mathrm{~F} 82$ & $4^{*}$ & 0×1C673D52FCD6E005D2A2B3D40EDCF & $2^{*}$ \\
\hline 0XAB5ED 9 & $14^{*}$ & 0x178CB7D5D6E322 & 2 & 0XE159BE 4A8763011C & 4 & 0x1C670773DF1678DF0A4336D3FE21E & 2 \\
\hline $0 \times 8 \mathrm{EFE} 15$ & $14^{*}$ & $0 \times 17641 \mathrm{C} 46 \mathrm{~F} 799 \mathrm{EE}$ & 2 & 0XE0A72F05397829CA & $4^{*}$ & 0X1C4D 4290F01337DEE39B3A7862BDE & 2 \\
\hline $0 \times 897 \mathrm{ECD}$ & $14^{*}$ & $0 \times 175 D 929 \mathrm{C} 3 \mathrm{C} 2 \mathrm{FC} 9$ & 2 & 0XE0A720FAC 6 F 829 CA & $4^{*}$ & 0x1C4BD3136A6DB6DB6DB351F3546E2 & 2 \\
\hline OXFFFFFC & $16^{*}$ & $0 \times 1733 \mathrm{~B} 8284238 \mathrm{~F} 1$ & 2 & $0 \times E 073 \mathrm{COEE} 938231 \mathrm{~F} 9$ & $4^{*}$ & 0x1C49777FF62E0B0DA1A4CDB58587F & $2^{*}$ \\
\hline $0 \times F 83 F 04$ & $16^{*}$ & 0x17255CA25B68E1 & $2^{*}$ & OXDF738B 7CF 7F 482E 4 & 4 & 0x1C4814DFE06ECB 4EB88D00BA934F2 & 2 \\
\hline
\end{tabular}

Table 1. Some numbers with reciprocals closest to numbers( $\left.{ }^{*}\right)$ and midpoints 
As usual in algorithms of this kind, each operation uses a fused multiply-add (not a separate multiplication and addition), all steps but the last are performed in round-to-nearest mode with additional exponent range precluding the possibility of intermediate overflow or underflow, and the last operation is done in the intended rounding mode and target precision.

Embedded in this algorithm is the computation of a very accurate reciprocal approximation $y_{3}$. Originally, in the design of algorithms of this kind, the correctness of the final rounding of $q$ was justified by a theorem whose precondition requires perfect rounding of $y_{3}$ [9], and only later was it noted by the present author that a relative error $y_{3}=\frac{1}{b}(1+\epsilon)$ for $|\epsilon|<2^{-p}$ suffices, which can be satisfied by a relatively weak error condition on $y_{2}$ and the analysis of a few special cases [3, 8]. However, if we are in a situation where $a=1$ we might consider, instead of using the entire sequence, unpicking the algorithm for reciprocation to be used directly, since its latency is shorter by 1 operation, and it uses only 9 floating-point operations instead of 14:

$$
\begin{array}{lll}
\text { 1. } & y_{0}=\operatorname{frcpa}(b) & \\
\text { 2. } & d=1-b y_{0} & \\
\text { 3. } & d_{2}=d d & d_{3}=d d+d \\
\text { 4. } & y_{1}=y_{0}+y_{0} d_{3} & d_{5}=d_{2} d_{2}+d \\
\text { 5. } & y_{2}=y_{0}+y_{1} d_{5} & \\
\text { 6. } & e=1-b y_{2} & \\
\text { 7. } & y=y_{2}+e y_{2} &
\end{array}
$$

Now the question of whether $y$ is always correctly rounded becomes critical. First we will consider round-tonearest. The initial approximation returned by frcpa will satisfy $y_{0}=\frac{1}{b}\left(1+\epsilon_{0}\right)$ for some $\left|\epsilon_{0}\right| \leq 2^{-8.886}$. A routine relative error analysis, assuming each rounding $r n(x)$ yields $x(1+\epsilon)$ for some $|\epsilon| \leq 2^{-64}$, shows that $y^{*}$, the value of $y$ before the last rounding, satisfies

$$
y^{*}=\frac{1}{b}(1+\epsilon)
$$

where $|\epsilon| \leq 2^{-123.37}$. Therefore, the only cases where incorrect rounding can occur are those closer than this relative distance to a midpoint. The potentially failing significands $b$ can be isolated by finding all $\left(2^{65}, 2^{64}\right)$-balanced factorizations $m b=2^{128}+d$ for integers $|d| \leq 24$ (since $24+1>2^{-123.37} / 2^{-128}$ ) and $m$ odd. The set of $b$ values that we need to consider are the following 134 (ordered in decreasing size, not according to their closeness to a midpoint):

OXFFFFFFFFFFFFFF OXFFFFFFFFFFFFFFD 0xFE421D63446A3B34 0xFBFC17DFEOBEFF04 0xFB940B119826E598 0xFB0089D7241D10FC 0xFA0BF7D05FBE82FC 0xF912590F016D6D04 0xF774DD7F912E1F54 OXF7444DFBF7B20EAC 0XF39EB657E24734AC OXF36EE790DE069D54 OXF286AD7943D79434 0xEDF09CCC53942014 0xEC4B058D0F7155BC 0XEC1CA6DB6D7BD 444 0XE775FF 856986AE74 0XE5CB972E5CB972E 4 0XE58469F0234F72C4 0xE511C4648E2332C4 0XE3FC771FE3B8FF1C
0XE318DE3C8E6370E4 0XE23B9711DCB88EE4 0XE159BE4A8763011C 0XDF738B7CF7F482E4 OXDEE256F712B7B894 0XDEE24908EDB7B894 0XDE86505A77F81B25 0XDE03D5F96C8A976C 0XDDFF059997C451E5 0xDB73060F0C3B6170 0xDB6DB6DB6DB6DB6C 0xDB6DA92492B6DB6C 0xDA92B6A4ADA92B6C 0xD9986492DD18DB7C 0xD72F32D1C0CC4094 0xD6329033D6329033 0xD5A004AE261AB3DC 0xD4D43A30F2645D7C 0xD33131D2408C6084 0xD23F53B88EADABB4 0xCCCE6669999CCCD0 0xCCCE 666666633330 0xCCCCCCCCCCCCCCD 0 0xCBC489A1DBB2F124 0xCB21076817350724 0xCAF92AC7A6F19EDC 0xC9A8364D41B26A0C 0xC687D6343EB1A1F4 0xC54EDD8E76EC6764 0xC4EC4EC362762764 0XC3FCF 61FE7B0FF3C 0XC3FCE9E018B0FF3C 0XC344F8A627C53D74 0xC27B1613D8B09EC4 0xC27B09EC27B09EC4 0xC07756F170EAFBEC 0xBDF3CD1B9E68E8D 4 0xBD 5EAF57ABD5EAF 4 0xBCA1AF286BCA1AF4 0xB9B501C68DD6D90C 0xB880B72F050B57FC 0xB85C824924643204 0xB7C8928A28749804 0xB7A481C71C43DDFC 0xB7938C6947D97303 0xB38A7755BB835F24 0xB152958A94AC54A4 0XAFF5757FABABFD5C OXAF 4D99ADFEFCAAFC 0XAF2B32F270835F04 0XAE235074CF5BAE64 0XAE0866F90799F954 0xADCC548E46756E64 0xAD5AB56AD5AB56AC 0xAD5AAA952AAB56AC 0xAB55AAD56AB55AAC 0xAAAAB55555AAAAAC 0xAAAAAAAAAAAAAAAC 0xAAAAA00000555554 0xA93CFF3E629F347D 0XA80555402AAA0154 0xA8054ABFD5AA0154 0XA7F94913CA4893D 4 0XA62E84F95819C3BC 0XA5889F09A0152C44 0XA4E75446CA6A1A44 0XA442B4F8DCDEF5BC 0xA27E096B503396EE 0x9E9B8FFFFFD8591C 0x9E9B8B0B23A7A6E 4 0x9E7C6B0C1CA79F1C 0X9DFC78A4EEEE4DCB 0x9C15954988E121AB 0x9A585968B4F4D2C4 0x99D0C486A0FAD481 0x99B831EEE01FB16C 0x990C8B8926172254 0x990825E0CD75297C 0x989E556CADAC2D7F 0x97DAD92107E19484 0x9756156041DBBA94 0X95C4C0A72F501BDC 0x94E1AE991B4B4EB4 0x949DE0B0664FD224 0×942755353AA9A094 0x9349AE0703CB65B4 0x92B6A4ADA92B6A4C 0x9101187A01C04E4C 0x907056B6E018E1B4 0x8E808E79E77A99C4 0x8F64655555317C3C 0x8E988B8B3BA3A624 0x8E05E117D9E786D5 0x8BEB067D130382A 4 0x8B679E2B7FB0532C 0x887C8B2B1F1081C4 0x8858CCDCA9E0F6C4 0x881BB1CAB40AE884 0x87715550DCDE29E4 0x875BDE4FE977C1EC 0x86F71861FDF38714 0x85DBEE9FB93EA864 0x8542A9A4D2ABD5EC 0x8542A150A8542A14 0x84BDA12F684BDA14 0X83AB6A090756D410 0x83AB6A06F8A92BF0 0x83A7B5D13DAE81B4 0x8365F2672F9341B4 0x8331C0CFE9341614 0x82A5F5692FAB4154 0x8140A05028140A04 0x8042251A9D6EF7FC

One can show by explicit computation that the algorithm works correctly on these values. It therefore rounds correctly on all values in round-to-nearest.

For directed rounding modes, the situation is less good. Once again the relative error condition gives rise to a set of test points, this time 227 of them. The algorithm works correctly on 220 of them, but not on floating-point numbers with one of the following 7 significands, the last of these representing exact powers of 2 , for which the true result is exactly representable. Cognoscenti who perform a back-ofenvelope calculation will not be surprised by the failure on exactly representable results, since correctness here would require $y_{2}$ already to be the correct result, which our relative error cannot quite guarantee.

$0 \times 8 c 82$ da588adc $64160 \times 84$ fdf 027 ef $813 f 7 b \quad 0 \times 827 b 9 b 8059090 a b 2$ $0 \times 80804020100804010 \times 80000800004000010 \times 8000000000000001$ $0 \times 8000000000000000$

This analysis indicates that the algorithm will produce correctly rounded results if the ambient rounding mode is known to be round-to-nearest, but will not always guarantee correct rounding in other rounding modes. Moreover, note that for the same reason, the 'inexact' flag will be incorrectly set in round-to-nearest mode in the special cases where $b$ is a power of 2. (As noted, the penultimate approximation $y_{2}$ cannot be the exact reciprocal in such cases, for otherwise we would obtain $e=0$ and correct rounding in all 
modes.) However, if this is considered important, it would be easy to detect and fix the problem with special case code without affecting overall latency.

\section{$7 \quad$ Feasibility study}

Although the previous sections show that the method is usefully applicable to some real problems, it's worth analyzing how practical the approach is likely to be in general. In attempting to use the method, three potential practical problems might arise

- Too many special points are isolated for further analysis to be feasible

- The factorization of some of the numbers is not feasible

- The distribution of prime factors is not feasible

We will not analyze the feasibility of factorization, since we do not understand the details of its implementation. We will however make the empirical observation that all factorizations for precisions up to $p=64$ seem to be very straightforward for PARI / GP, taking a fraction of a second, while those for $p=113$ usually take several seconds and, exceptionally, minutes.

\section{Average density of balanced factorizations}

It is not difficult to see that "on average" we obtain a fairly modest number of balanced factorizations per value examined. First note that the number of $(A, B)$-balanced products of numbers $\leq n$ is the number of lattice points contained both within the rectangle $0 \leq x \leq A, 0 \leq y \leq B$ and under the curve $x y=n$. We can get a good estimate by ignoring "edge effects" and just considering the plane area, integrating to obtain:

$$
C(n)=n\left(1+\ln \left(\frac{A B}{n}\right)\right)
$$

Differentiating with respect to $n$ yields the expected density, i.e. the average number of $(A, B)$-balanced product representations of a number close to $n$ :

$$
D(n)=\ln (A B / n)
$$

Of course, these gross averages do not reflect smallscale fluctuations. Nevertheless, the agreement is fairly good with some empirical results obtained by sampling. In the following table, we examine the density of $\left(2^{p}, 2^{p}\right)$ balanced products for several $p$, looking in each case at 31 regions close to $\frac{k+1 / 2}{32} 2^{2 p}$ for $0 \leq k \leq 31$ and sampling 1024 successive points in each. The final figures at the bottom give the mean value. This indicates how accurate the sampling process is on average; perfectly representative sampling would give exactly 1 here. (We avoid sampling at $\frac{k}{32} 2^{p}$ because that would lead to strong correlations between the sets of numbers at different $k$.)

\begin{tabular}{l|l|l|l}
$\ln \left(2^{2 p} / n\right)$ & $p=24$ & $p=53$ & $p=64$ \\
\hline 4.1588 & 4.4785 & 4.6835 & 3.3300 \\
3.0602 & 2.8496 & 5.6621 & 3.2734 \\
2.5494 & 2.4570 & 2.7070 & 2.2753 \\
2.2129 & 2.0332 & 2.2421 & 2.2089 \\
1.9616 & 2.0000 & 1.6953 & 2.3417 \\
1.7609 & 1.9101 & 1.5664 & 1.5585 \\
1.5939 & 1.5742 & 1.9140 & 1.2128 \\
1.4508 & 1.3632 & 1.4765 & 1.5625 \\
1.3256 & 1.3144 & 1.0839 & 1.2558 \\
1.2144 & 1.2050 & 1.2187 & 1.2890 \\
1.1143 & 1.0175 & 1.0996 & 1.4296 \\
1.0233 & 1.0273 & 0.9335 & 0.9687 \\
0.9400 & 0.7539 & 0.9062 & 0.8828 \\
0.8630 & 0.7636 & 0.8613 & 0.8789 \\
0.7915 & 0.6875 & 0.7187 & 0.6875 \\
0.7248 & 0.6933 & 0.6621 & 0.7832 \\
0.6623 & 0.6621 & 0.5976 & 0.7656 \\
0.6035 & 0.5878 & 0.5468 & 0.6445 \\
0.5479 & 0.5546 & 0.6210 & 0.5683 \\
0.4953 & 0.4941 & 0.5136 & 0.6289 \\
0.4453 & 0.4394 & 0.3847 & 0.3652 \\
0.3976 & 0.3984 & 0.4453 & 0.4277 \\
0.3522 & 0.3417 & 0.3476 & 0.3242 \\
0.3087 & 0.3203 & 0.2890 & 0.3593 \\
0.2670 & 0.2382 & 0.2285 & 0.2773 \\
0.2270 & 0.2480 & 0.2070 & 0.3007 \\
0.1885 & 0.1347 & 0.2207 & 0.2148 \\
0.1515 & 0.1347 & 0.1640 & 0.1562 \\
0.1158 & 0.0839 & 0.0976 & 0.1015 \\
0.0813 & 0.0917 & 0.1054 & 0.0761 \\
0.0480 & 0.0449 & 0.0371 & 0.0527 \\
0.0157 & 0.0078 & 0.0078 & 0.0156 \\
\hline 1.0000 & 0.9660 & 1.0701 & 0.9755 \\
& & &
\end{tabular}

So much for the average case. What about the worst case? This seems a more difficult question to address theoretically, but in the next section we will show how to obtain a pessimistic upper bound.

\section{Feasibility of distribution algorithm}

Although the final number of values produced depends on the number of balanced factorizations, the process by which the balanced factorizations are enumerated involves examination of many dead-end paths, so the runtime of the distribution process may be very large relative to the final number of possibilities produced. A reasonable, though pessimistic, bound on the runtime of the distribution algorithm for a value $n$ is $d(n)$, the total number of divisors of $n$, regardless of balance. For even without early cutoffs owing to range limitations, the algorithm cannot examine, given

$$
n=\Pi_{i=1}^{i=k} p_{i}^{\alpha_{i}}
$$

more than

$$
d(n)=\Pi_{i=1}^{i=k}\left(1+\alpha_{i}\right)^{n}
$$


possibilities, since each factor $p_{i}^{\alpha_{i}}$ can, without range cutoffs, be distributed in $1+\alpha_{i}$ ways.

It is well known that the average number of divisors $d(n)$ of a number near $n$ is approximately $d(n)=\ln (n)$. This can easily be derived using the same sort of argument as we used above for balanced products [2]. This suggests that on average, the distribution process will not have many cases to examine; even for quad precision, we have $n \leq 2^{230}$ and so $\ln (n) \leq 160$.

What about the worst case? The number of divisors of a number can be much larger than $\ln (n)$. In fact [2], almost all numbers (in a precise sense) have about $\ln (n)^{\ln (2)}$ divisors, with the larger overall average of $\ln (n)$ resulting from a small proportion of numbers with many more divisors. Asymptotically, it is known [2] that $d(n)$ has an upper limit of exactly $2^{\ln (n) / \ln (\ln (n))}$, or more precisely, that if $\epsilon>0$ then $d(n)<2^{(1+\epsilon) \ln (n) / \ln (\ln (n))}$ for all sufficiently large $n$, while $d(n)>2^{(1-\epsilon) \ln (n) / \ln (\ln (n))}$ for infinitely many $n$.

This asymptotic limit needs refinement to be useful to us for the concrete ranges we are interested in. We can obtain a more refined estimate of the maximum $d(n)$ for all $n$ below some limit $N$ we are interested in as follows. The key to efficient search is to seek the minimal $n$ with the maximal number of divisors possible for $n \leq N$. The minimality constraint forces strong patterns onto the prime factorization. Suppose that $n$ has the following prime factorization:

$$
n=\prod_{i=1}^{i=k} p_{i}^{\alpha_{i}}
$$

Let $p_{i}<p_{j}$ be two primes (not necessarily appearing with nonzero index in the above factorization) such that $p_{i}^{\beta}<p_{j}<p_{i}^{\beta+1}$ for some nonnegative integer $\beta$. Then it is easy to see that if $n$ has the minimality property, the following relationships hold between the $\alpha$ 's:

$$
\beta \alpha_{j} \leq \alpha_{i} \leq(\beta+1) \alpha_{j}+2 \beta
$$

For if the first inequality failed we could get a smaller number with at least as many divisors by replacing $p_{i}^{\alpha_{i}} p_{j}^{\alpha_{j}}$ with $p_{i}^{\alpha_{i}+\beta} p_{j}^{\alpha_{j}-1}$, while if the second inequality failed we could likewise replace it with $p_{i}^{\alpha_{i}-(\beta+1)} p_{j}^{\alpha_{j}+1}$.

This observation includes the case where $p_{j}$ is the first prime beyond those appearing in the factorization, and in this case $\alpha_{i} \leq 2 \beta$. For example, if $17^{\alpha}$ appears in the factorization, so must $3^{2 \alpha}$ and $2^{4 \alpha}$, while if no power of 17 appears in the factorization then the highest possible power of 2 appearing is $2^{8}$, and the highest power of 3 is $3^{6}$. Note in particular that the factorization of the minimal $n$ must contain the first $k$ consecutive primes without gaps, for some $k$.

These observations cut down the search space dramatically enough that we can easily perform an exhaustive search for the precise worst numbers up to quite large values, say $2^{3000}$. The following table shows, for various values of $p$ up to 230 , the minimal $n \leq 2^{p}$ with the largest number of divisors possible in that range. For each such $n$, we show $\log _{2}(n)$ and $\log _{2}(d(n))$ (where $d(n)$ is the number of divisors of $n$ ), as well as the ratio with the expected limit superior $r(n)=\log _{2}(d(n)) /(\ln (n) / \ln (\ln (n)))$ and the ac-

\begin{tabular}{|c|c|c|c|c|}
\hline$p$ & $\log _{2}(n)$ & $\log _{2}(d(n))$ & $r(n)$ & Factorization of that worst $n$ \\
\hline 10 & 9.71 & 5.00 & 1.416 & $2^{3} 357$ \\
\hline 20 & 19.45 & 7.90 & 1.525 & $2^{4} 3^{2} 5 \cdots 13$ \\
\hline 30 & 29.45 & 10.39 & 1.535 & $2^{6} 3^{3} 5^{2} 7 \cdots 17$ \\
\hline 40 & 39.80 & 12.71 & 1.528 & $2^{6} 3^{4} 5^{2} \quad \ldots \ldots 23$ \\
\hline 50 & 49.84 & 14.75 & 1.512 & $2^{5} 3^{3} 5^{2} 7^{2} 11 \cdots 31$ \\
\hline 60 & 59.96 & 16.71 & 1.498 & $2^{6} 3^{4} 5^{3} 7^{2} 11 \cdots 37$ \\
\hline 70 & 69.42 & 18.49 & 1.488 & $2^{7} 3^{4} 5^{2} 7^{2} \quad 11 \ldots 43$ \\
\hline 80 & 79.88 & 20.33 & 1.474 & $2^{8} 3^{5} 5^{3} 7^{2} 11 \ldots 47$ \\
\hline 90 & 89.90 & 22.07 & 1.463 & $2^{8} 3^{4} 5^{3} 7^{2} \quad 11 \ldots 59$ \\
\hline 100 & 99.88 & 23.75 & 1.453 & $2^{7} 3^{5} 5^{3} 7^{2} 1^{2} \quad 13 \cdots 61$ \\
\hline 110 & 109.64 & 25.33 & 1.443 & $2^{8} 3^{5} 5^{3} 7^{2} 11 \cdots 71$ \\
\hline 120 & 119.87 & 26.97 & 1.435 & $2^{7} 3^{6} 5^{3} 7^{2}{ }_{11^{2}}^{2} 13 \cdots 73$ \\
\hline 130 & 129.87 & 28.56 & 1.427 & $2^{7} 3^{6} 5^{3} 7^{2} \quad 11^{2} 1^{2} \quad 17 \cdots 79$ \\
\hline 140 & 139.99 & 30.12 & 1.420 & $2^{10} 3^{5} 5^{4} 7^{2} 11^{2} 13^{2} 17 \cdots 83$ \\
\hline 150 & 149.74 & 31.66 & 1.416 & $2^{9} 3^{5} 5^{3} 7^{2} 11^{2} 13^{2} 17 \cdots 97$ \\
\hline 160 & 159.79 & 33.14 & 1.408 & $2^{8} 3^{6} 5^{3} 7^{3} 11^{2} 1^{2} \quad 17 \cdots 101$ \\
\hline 170 & 169.83 & 34.66 & 1.404 & $2^{9} 3^{5} 5^{3} 7^{2} 1_{11^{2}} 13^{2} \quad 17 \cdots 107$ \\
\hline 180 & 179.99 & 36.14 & 1.398 & $2^{8} 3^{6} 5^{3} 7^{3} 1_{11^{2}} 13^{2} 17 \ldots 109$ \\
\hline 190 & 189.82 & 37.56 & 1.393 & $2^{9} 3^{5} 5^{4} 7^{2} 11^{2} 13^{2} 17^{2} 19 \cdots 113$ \\
\hline 200 & 199.88 & 39.02 & 1.388 & $2^{10} 3^{6} 5^{3} 7^{3}{ }_{11^{2}}^{2} 13^{2} 1^{2} \quad 19 \cdots 127$ \\
\hline 210 & 209.93 & 40.43 & 1.383 & $2^{10} 3^{6} 5^{3} 7^{3} 1^{2} 13^{2} 17 \cdots 137$ \\
\hline 220 & 219.87 & 41.83 & 1.379 & $2^{8} 3^{5} 5^{4} 7^{3} 11^{2} 13^{2} 17^{2} 19 \cdots 139$ \\
\hline 230 & 229.92 & 43.21 & 1.375 & $2^{10} 3^{5} 5^{3} 7^{3} 11^{2} 13^{2} 17 \cdots 151$ \\
\hline
\end{tabular}
tual factorization of $n$.

We can see that even for double-extended precision, the number of factorizations that could possibly need to be examined is about $2^{28}$. Although a fairly large number, this is definitely feasible. (And of course in practice such cases are exceptional and not all factorizations would be examined.) For quad precision, on the other hand, it is entirely possible for the search to be infeasible. We have not yet encountered this phenomenon in practice, however.

Note that $d(n)$ also gives an upper bound to the number of balanced factorizations. It is, of course, pessimistic, but testing on some of the values above suggests that the the number of balanced factorizations is a reasonable proportion (say 10\%) of the total number of divisors. Naturally, it would be better to refine all these estimates to consider only numbers very close to the powers of 2 , which is what we are really interested in.

The special numbers that we searched for above are particular cases of highly composite numbers [13]. For a detailed survey of the subject see [10], while Achim Flammenkamp's Web page seems to give a more efficient algorithm for generating $\mathrm{HCNs}$ :

http://wwwhomes.uni-bielefeld.de/achim/highly.html

The sequence of highly composite numbers is A002182 in Sloane's Encyclopedia of Integer Sequences.

\section{Extension to reciprocal square root}

It is interesting to note that a similar factor distribution technique can be used to attempt to find exceptional cases 
for the reciprocal square root. In this case, we seek floatingpoint numbers or midpoints $w$ and floating-point numbers $y$ such that

$$
\frac{\left|w-\frac{1}{\sqrt{y}}\right|}{\left|\frac{1}{\sqrt{y}}\right|}
$$

is small. We can rewrite this as:

$$
\left|\sqrt{y}\left(w-\frac{1}{\sqrt{y}}\right)\right|=|w \sqrt{y}-1|
$$

In the critical cases where $w \sqrt{y}-1$ is very small, then $w \sqrt{y}+1$ is almost exactly 2 and so:

$$
|w \sqrt{y}-1|=\frac{\left|w^{2} y-1\right|}{|w \sqrt{y}+1|} \approx \frac{\left|w^{2} y-1\right|}{2}
$$

Once again, let us scale the values $w$ and $y$ to integers $m$ and $b$ :

$$
\begin{array}{ll}
y=2^{e_{y}} b & 2^{p-1} \leq b<2^{p} \\
w=2^{e_{w}} m & 2^{p} \leq m<2^{p+1}
\end{array}
$$

and then the distance we are interested in is then:

$$
\frac{\left|m^{2} b-2^{q}\right|}{2^{q+1}}
$$

where $q=-\left(2 e_{w}+e_{y}\right)$. So we seek cases where $d=$ $m^{2} b-2^{q}$ is as small as possible. Keeping in mind the range restrictions, we see that $2^{3 p-1} \leq m^{2} b<2^{3 p+2}$. As with simple reciprocals, it is impossible to come very close to the extremal powers of 2 , but we do now need to consider two cases, $q=3 p$ and $q=3 p+1$.

The reciprocal square root function is of some theoretical interest because it seems prima facie possible that $d=m^{2} b-2^{q}$ could be very small, perhaps even \pm 1 , yet no precisions where it is much smaller than $2^{p}$ have ever been found, and one might expect on naive statistical grounds that it is unlikely. (We only have $2^{2 p}$ different choices of pairs $m$ and $b$, and are scattering the resulting $m^{2} b$ 's somehow over an interval of size about $2^{3 p}$.) Li [7] proves that assuming the $\mathrm{ABC}$ conjecture from number theory holds, the distance is indeed of order $2^{p}$ for all sufficiently large $p$. Even if the ABC conjecture were proven, however, it's not clear whether it would be possible to constructivize the proof in order to obtain useful bounds for specific precisions. Iordache and Matula [4] observe that $d=1$ is impossible in general, allowing the accuracy required to be lowered slightly, but add that 'trying to lower it is not an easy problem, even for a fixed $p$ '. Although the present work does not touch the general case, and nor can it fully bridge the gap between expected and provable bounds, it does allow us quite easily to improve the provable bound for the typical $p$ we are interested in by a reasonable factor.
We can take over the prime distribution function with little change. The only difference is that we now need to distribute the prime factors among $m^{2} b$. This has the immediate consequence that only even powers of primes can be allocated to the $m^{2}$ part, and so any prime appearing to an odd power in the prime factorization of $2^{q}+d$ must be allocated at least once to $b$. This is almost always enough to render the distribution immediately impossible. We have made some searches for double-extended precision $(p=64)$ and quad precision $(p=113)$. For double-extended, we have shown that $d \leq 1024$ is impossible, and it would be easy to continue the search much further. For quad precision, the cost of factoring numbers is now a serious bottleneck, with a single number sometimes taking a day of CPU time and one of the factorizations for the $d=6$ case apparently defeating factorization in a reasonable time. Nevertheless we have at least shown that $d<6$ is impossible, which represents some improvement. For smaller precisions, it seems likely that other algorithms based on an (intelligent) exhaustive analysis of the whole space of significands would be more efficient. For example Lang and Muller [5] have performed a complete analysis of the double-precision case $p=53$ (and found that the minimal distance is about $2^{-110}$ ).

\section{Conclusion}

The methods described here allow reasonably effective isolation of the 'worst cases' for the reciprocal function. This opens the way to correctness proofs of reciprocal algorithms using the same kind of two-part approach used by Cornea [1] for square roots. In the absence of new theoretical advances, the method described may also be the best available means of improving the difficulty bounds on the reciprocal square root functions for larger precisions. Although our method has feasibility problems for the extreme case of quad-precision reciprocal square roots, it would be possible to explore alternative factoring algorithms. The numbers we are interested in factoring are very close (in relative terms) to powers of 2, so it is possible that algorithms such as the Special Number Field Sieve (SNFS) would give much better results.

\section{Acknowledgements}

The author is grateful to the anonymous referees, who made a number of excellent suggestions, and pointed out connections of which the author had been unaware.

\section{References}

[1] M. Cornea-Hasegan. Proving the IEEE correctness of iterative fbating-point square root, divide 
and remainder algorithms. Intel Technology Journal, 1998-Q2:1-11, 1998. Available on the Web as http: //developer.intel.com/technology/ itj/q21998/articles/art_3.htm.

[2] G. H. Hardy and E. M. Wright. An Introduction to the Theory of Numbers. Clarendon Press, 5th edition, 1979.

[3] J. Harrison. Formal verifi cation of IA-64 division algorithms. In M. Aagaard and J. Harrison, editors, Theorem Proving in Higher Order Logics: 13th International Conference, TPHOLs 2000, volume 1869 of Lecture Notes in Computer Science, pages 234-251. Springer-Verlag, 2000.

[4] C. Iordache and D. W. Matula. On infi nitely precise rounding for division, square root, reciprocal and square root reciprocal. In I. Koren and P. Kornerup, editors, Proceedings, 14th IEEE symposium on on computer arithmetic, pages 233-240, Adelaide, Australia, 1999. IEEE Computer Society. See also Technical Report 99-CSE-1, Southern Methodist University.

[5] T. Lang and J.-M. Muller. Bounds on runs of zeros and ones for algebraic functions. Research Report 4045, INRIA, 2000.

[6] V. Lefèvre and J.-M. Muller. Worst cases for correct rounding of the elementary functions in double precision. Research Report 4044, INRIA, 2000.

[7] R.-C. Li. The ABC conjecture and correctly rounded reciprocal square root. Preprint, 2002.

[8] P. Markstein. IA-64 and Elementary Functions: Speed and Precision. Prentice-Hall, 2000.

[9] P. W. Markstein. Computation of elementary functions on the IBM RISC System/6000 processor. IBM Journal of Research and Development, 34:111-119, 1990.

[10] J.-L. Nicholas. On highly composite numbers. In Ramanujan Revisited: Proceedings of the Centenery Conference, pages 215-244. Academic Press, 1988.

[11] M. Parks. Number-theoretic test generation for directed rounding. IEEE Transactions on Computers, 49:651-658, 2000.

[12] V. Pratt. Every prime has a succinct certifi cate. SIAM Journal of Computing, 4:214-220, 1975.

[13] S. Ramanujan. Highly composite numbers. Proceedings of the London Mathematical Society, 14:347-409, 1915.

[14] P. T. P. Tang. Testing computer arithmetic by elementary number theory. Preprint MCS-P84-0889, Mathematics and Computer Science Division, Argonne National Labs, 1989. 